\title{
Stochastic differential delay equations with Markovian switching
}

\author{
XUERONG MAO $^{1}$, ALEXANDER MATASOV ${ }^{2}$ and \\ ALEKSEY B. PIUNOVSKIY ${ }^{3}$ \\ ${ }^{1}$ Department of Statistics and Modelling Science, University of Strathclyde, Glasgow G1 1 XH, \\ UK.Email:xuerong@stams.strath.ac.uk \\ ${ }^{2}$ Faculty of Mechanics and Mathematics, Moscow Lomonosov State University, Russia. \\ E-mail: amatasov@nw.math.msu.su \\ ${ }^{3}$ Moscow State Institute of Physics and Technology, Moscow 125206, Russia. \\ E-mail:pi@dep11.ifftr.msk.su
}

In this paper we discuss stochastic differential delay equations with Markovian switching. These can be regarded as the result of several stochastic differential delay equations switching among each other according to the movement of a Markov chain. One of the main aims of this paper is to investigate the exponential stability of the equations.

Keywords: Brownian motion; delay equation; generalized Itô's formula; Lyapunov exponent; Markov chain

\section{Introduction}

Stochastic modelling has come to play an important role in many branches of science and industry. An area of particular interest has been the automatic control of stochastic systems, with consequent emphasis being placed on the analysis of stability in stochastic models, and we here mention Arnold (1972), Has'minskii (1981), Kolmanovskii and Myshkis (1992), Kolmanovskii and Nosov (1986), Ladde and Lakshmikantham (1980), Mao (1991; 1994) and Mohammed (1986) among others. There has been little work on the stability of stochastic differential delay equations with Markovian switching, although there are several papers on the stability of stochastic differential equations with Markovian switching, for example Basak et al. (1996), Ghosh et al. (1993) and Skorohod (1989).

In this paper we consider a stochastic differential delay equation with Markovian switching of the form

$$
\mathrm{d} x(t)=f(x(t), x(t-\tau), t, r(t)) \mathrm{d} t+g(x(t), x(t-\tau), t, r(t)) \mathrm{d} w(t),
$$

where $r(t)$ is a Markov chain taking values on $S=\{1,2, \ldots, N\}$. This equation can be regarded as the result of the $N$ equations

$$
\mathrm{d} x(t)=f(x(t), x(t-\tau), t, i) \mathrm{d} t+g(x(t), x(t-\tau), t, i) \mathrm{d} w(t), \quad 1 \leqslant i \leqslant N,
$$


switching among each other according to the movement of the Markov chain. In Section 2 we shall quickly establish the theorem for the existence and uniqueness of the solution of the equation and cite the generalized Itô formula. We shall then discuss the mean square exponential stability of linear stochastic differential delay equations with Markovian switching in Section 3, and generalize the results to the $p$ th moment exponential stability for equation (1.1) in Section 4. The almost sure exponential stability will be studied in Section 5. Finally, we give some illustrative examples in Section 6.

\section{Stochastic differential delay equations with Markovian switching}

Throughout this paper, unless otherwise specified, we let $\left(\Omega, \mathscr{F},\left\{\mathscr{F}_{t}\right\}_{t \geqslant 0}, P\right)$ be a complete probability space with a filtration $\left\{\mathscr{F}_{t}\right\}_{t \geqslant 0}$ satisfying the usual conditions (i.e. it is right continuous and $\mathscr{F}_{0}$ contains all $P$-null sets). Let $w(t)=\left(w_{1}(t), \ldots, w_{m}(t)\right)^{\mathrm{T}}$ be an $m$ dimensional Brownian motion defined on the probability space. Let $\tau>0$ and $C\left([-\tau, 0] ; \mathbb{R}^{n}\right)$ denote the family of continuous functions $\varphi$ from $[-\tau, 0]$ to $\mathbb{R}^{n}$ with the norm $\|\varphi\|=\sup _{\tau \leqslant \theta \leqslant 0}|\varphi(\theta)|$, where $|\cdot|$ is the Euclidean norm in $\mathbb{R}^{n}$. If $A$ is a vector or matrix, its transpose is denoted by $A^{\mathrm{T}}$. If $A$ is a matrix, its trace norm is denoted by $|A|=\sqrt{\operatorname{tr}\left(A^{\mathrm{T}} A\right)}$ while its operator norm is denoted by $\|A\|=\sup \{|A x|:|x|=1\}$ (without any confusion with $\|\varphi\|)$. If $A$ is a symmetric matrix, denote by $\lambda_{\max }(A)$ and $\lambda_{\min }(A)$ its largest and smallest eigenvalue, respectively. Denote by $C_{\mathscr{F}_{0}}^{\mathrm{b}}\left([-\tau, 0] ; \mathbb{R}^{n}\right)$ the family of all bounded, $\mathscr{F}_{0^{-}}$ measurable, $C\left([-\tau, 0] ; \mathbb{R}^{n}\right)$-valued random variables. If $x(t)$ is a continuous $\mathbb{R}^{n}$-valued stochastic process on $t \in[-\tau, \infty)$, we let $x_{t}=\{x(t+\theta):-\tau \leqslant \theta \leqslant 0\}$ for $t \geqslant 0$, which is regarded as a $C\left([-\tau, 0] ; \mathbb{R}^{n}\right)$-valued stochastic process.

Let $r(t), t \geqslant 0$, be a right-continuous Markov chain on the probability space taking values in a finite state space $S=\{1,2, \ldots, N\}$ with generator $\Gamma=\left(\gamma_{i j}\right)_{N \times N}$ given by

$$
P\{r(t+\Delta)=j \mid r(t)=i\}= \begin{cases}\gamma_{i j} \Delta+o(\Delta), & \text { if } i \neq j, \\ 1+\gamma_{i i} \Delta+o(\Delta), & \text { if } i=j,\end{cases}
$$

where $\Delta>0$. Here $\gamma_{i j} \geqslant 0$ is the transition rate from $i$ to $j$ if $i \neq j$, while

$$
\gamma_{i i}=-\sum_{j \neq i} \gamma_{i j}
$$

We assume that the Markov chain $r(\cdot)$ is independent of the Brownian motion $w(\cdot)$. It is known that almost every sample path of $r(t)$ is a right-continuous step function with a finite number of simple jumps in any finite subinterval of $\mathbb{R}_{+}(:=[0, \infty))$.

Consider a stochastic differential delay equation with Markovian switching of the form

$$
\mathrm{d} x(t)=f(x(t), x(t-\tau), t, r(t)) \mathrm{d} t+g(x(t), x(t-\tau), t, r(t)) \mathrm{d} w(t)
$$

on $t \geqslant 0$, with initial data $x_{0}=\xi \in C_{\mathscr{F}_{0}}^{\mathrm{b}}\left([-\tau, 0] ; \mathbb{R}^{n}\right)$, where

$$
f: \mathbb{R}^{n} \times \mathbb{R}^{n} \times \mathbb{R}_{+} \times S \rightarrow \mathbb{R}^{n} \quad \text { and } g: \mathbb{R}^{n} \times \mathbb{R}^{n} \times \mathbb{R}_{+} \times S \rightarrow \mathbb{R}^{n \times m} .
$$


We impose a hypothesis:

(H1) Both $f$ and $g$ satisfy the local Lipschitz condition and the linear growth condition. That is, for each $k=1,2, \ldots$, there is an $h_{k}>0$ such that $|f(x, y, t, i)-f(\bar{x}, \bar{y}, t, i)|+|g(x, y, t, i)-g(\bar{x}, \bar{y}, t, i)| \leqslant h_{k}(|x-\bar{x}|+|y-\bar{y}|)$ for all $t \geqslant 0, i \in S$ and those $x, y, \bar{x}, \bar{y} \in \mathbb{R}^{n}$ with $|x| \vee|y| \vee|\bar{x}| \vee|\bar{y}| \leqslant k$; and there is, moreover, an $h>0$ such that

$$
|f(x, y, t, i)|+|g(x, y, t, i)| \leqslant h(1+|x|+|y|)
$$

for all $x, y \in \mathbb{R}^{n}, t \geqslant 0$ and $i \in S$.

Theorem 2.1. Under hypothesis (H1), equation (2.1) has a unique continuous solution $x(t)$ on $t \geqslant-\tau$. Moreover, for every $p>0$,

$$
\mathrm{E}\left[\sup _{-\tau \leqslant s \leqslant t}|x(s)|^{p}\right]<\infty \quad \text { on } t \geqslant 0 .
$$

Proof. It should be pointed out that the reason why we let the initial data be bounded in this paper is just for the study of $p$ th moment stability; for the existence and uniqueness of the solution we only require the initial data to be $L^{2}$.

Existence and uniqueness. Let us introduce some new notation. For any stopping time $\rho$, denote by $L_{\mathscr{F}_{\rho}}^{2}\left([-\tau, 0] ; \mathbb{R}^{n}\right)$ the family of all $\mathscr{F}_{\rho}$-measurable $C\left([-\tau, 0] ; \mathbb{R}^{n}\right)$-valued random variables $\xi$ such that $\mathrm{E}\|\xi\|^{2}<\infty$. Obviously, $C_{\mathscr{F}_{0}}^{\mathrm{b}}\left([-\tau, 0] ; \mathbb{R}^{n}\right) \subset L_{\mathscr{F}_{0}}^{2}\left([-\tau, 0] ; \mathbb{R}^{n}\right)$. Let $T>0$ be arbitrary. It is sufficient to show that equation (2.1) has a unique solution on $[-\tau, T]$. It is known (see Skorohod 1989) that there is a sequence $\left\{\tau_{k}\right\}_{k \geqslant 0}$ of stopping times such that $0=\tau_{0}<\tau_{1}<\ldots<\tau_{k} \rightarrow \infty$ and $r(t)$ is constant on every interval $\left[\tau_{k}, \tau_{k+1}\right)$, that is, for every $k \geqslant 0$

$$
r(t)=r\left(\tau_{k}\right) \quad \text { on } \tau_{k} \leqslant t<\tau_{k+1} .
$$

We first consider equation (2.1) on $t \in\left[0, \tau_{1} \wedge T\right]$, which becomes

$$
\mathrm{d} x(t)=f(x(t), x(t-\tau), t, r(0)) \mathrm{d} t+g(x(t), x(t-\tau), t, r(0)) \mathrm{d} w(t),
$$

with initial data $x_{0}=\xi \in C_{\mathscr{T}_{0}}^{\mathrm{b}}\left([-\tau, 0] ; \mathbb{R}^{n}\right) \subset L_{\mathscr{T}_{0}}^{2}\left([-\tau, 0] ; \mathbb{R}^{n}\right)$. By the existence-anduniqueness theorem of stochastic differential delay equations (see Mao 1994; 1997; or Mohammed 1986) we know that equation (2.3) has a unique continuous solution on $\left[-\tau, \tau_{1} \wedge T\right]$ which has the property that

$$
\mathrm{E}\left[\sup _{-\tau \leqslant s \leqslant \tau_{1} \wedge T}|x(s)|^{2}\right]<\infty .
$$

We next consider equation (2.1) on $t \in\left[\tau_{1} \wedge T, \tau_{2} \wedge T\right]$, which becomes

$$
\mathrm{d} x(t)=f\left(x(t), x(t-\tau), t, r\left(\tau_{1} \wedge T\right)\right) \mathrm{d} t+g\left(x(t), x(t-\tau), t, r\left(\tau_{1} \wedge T\right)\right) \mathrm{d} w(t),
$$

with initial data $x_{\tau_{1} \wedge T} \in L_{\mathscr{T}_{\tau} \wedge T}^{2}\left([-\tau, 0] ; \mathbb{R}^{n}\right)$ given by the solution of equation (2.3). Again we know that equation (2.4) has a unique continuous solution on $\left[\tau_{1} \wedge T-\tau, \tau_{2} \wedge T\right]$. Repeating 
this procedure, we see that equation (2.1) has a unique solution $x(t)$ on $[-\tau, T]$. Since $T$ is arbitrary, the existence and uniqueness have been proved.

Moment estimation. To show (2.2), we note from Hölder's inequality that

$$
\left(\mathrm{E}\left[\sup _{-\tau \leqslant s \leqslant t}|x(s)|^{p}\right]\right)^{\frac{1}{p}} \leqslant\left(\mathrm{E}\left[\sup _{-\tau \leqslant s \leqslant t}|x(s)|^{2}\right]\right)^{\frac{1}{2}} \quad \text { if } 0<p<2 .
$$

So we only need to prove (2.2) in the case of $p \geqslant 2$. For each positive integer $k$, define a stopping time

$$
\rho_{k}=\inf \{t \geqslant 0:|x(t)| \geqslant k\}
$$

(as usual we set inf $\varnothing=\infty$ ). Clearly, $\rho_{k} \rightarrow \infty$ almost surely as $k \rightarrow \infty$. Again let $T>0$ be arbitrary. For any $t \in[0, T]$ and $k \geqslant 1$, by Hölder's inequality, Theorem 1.7.2 of Mao (1997) and the linear growth condition, we can show that

$$
\mathrm{E}\left[\sup _{0 \leqslant s \leqslant t}\left|x\left(s \wedge \rho_{k}\right)\right|^{p}\right] \leqslant 3^{p-1} \mathrm{E}|\xi(0)|^{p}+C \mathrm{E} \int_{0}^{t}\left(1+\left|x\left(s \wedge \rho_{k}\right)\right|^{p}+\left|x\left(s \wedge \rho_{k}-\tau\right)\right|^{p}\right) \mathrm{d} s,
$$

where $C$ is a positive constant dependent on $p, T$ and $h$ only. Consequently,

$$
\begin{aligned}
\mathrm{E}\left[\sup _{-\tau \leqslant s \leqslant t}\left|x\left(s \wedge \rho_{k}\right)\right|^{p}\right] & \leqslant \mathrm{E}\|\xi\|^{p}+\mathrm{E}\left[\sup _{0 \leqslant s \leqslant t}\left|x\left(s \wedge \rho_{k}\right)\right|^{p}\right] \\
& \leqslant\left(3^{p-1}+1\right) \mathrm{E}\|\xi\|^{p}+C T+2 C \int_{0}^{t} \mathrm{E}\left[\sup _{-\tau \leqslant s \leqslant u}\left|x\left(s \wedge \rho_{k}\right)\right|^{p}\right] \mathrm{d} u .
\end{aligned}
$$

An application of the Gronwall inequality implies

$$
\mathrm{E}\left[\sup _{-\tau \leqslant s \leqslant T}\left|x\left(s \wedge \rho_{k}\right)\right|^{p}\right] \leqslant \mathrm{e}^{2 C T}\left[\left(3^{p-1}+1\right) \mathrm{E}\|\xi\|^{p}+C T\right] .
$$

Letting $k \rightarrow \infty$, we obtain that

$$
\mathrm{E}\left[\sup _{-\tau \leqslant s \leqslant T}|x(s)|^{p}\right] \leqslant \mathrm{e}^{2 C T}\left[\left(3^{p-1}+1\right) \mathrm{E}\|\xi\|^{p}+C T\right] .
$$

and the required assertion (2.2) follows. The proof is complete.

Let $C^{2,1}\left(\mathbb{R}^{n} \times \mathbb{R}_{+} \times S ; \mathbb{R}_{+}\right)$denote the family of all non-negative functions $V(x, t, i)$ on $\mathbb{R}^{n} \times \mathbb{R}_{+} \times S$ which are twice continuously differentiable in $x$ and once differentiable in $t$. If $V \in C^{2,1}\left(\mathbb{R}^{n} \times \mathbb{R}_{+} \times S ; \mathbb{R}_{+}\right)$, define an operator $L V$ from $\mathbb{R}^{n} \times \mathbb{R}^{n} \times \mathbb{R}_{+} \times S$ to $\mathbb{R}$ by

$$
\begin{aligned}
L V(x, y, t, i)= & V_{t}(x, t, i)+V_{x}(x, t, i) f(x, y, t, i) \\
& +\frac{1}{2} \operatorname{tr}\left[g^{\mathrm{T}}(x, y, t, i) V_{x x}(x, t, i) g(x, y, t, i)\right]+\sum_{j=1}^{N} \gamma_{i j} V(x, t, j),
\end{aligned}
$$

where 


$$
\begin{gathered}
V_{t}(x, t, i)=\frac{\partial V(x, t, i)}{\partial t}, \quad V_{x}(x, t, i)=\left(\frac{\partial V(x, t, i)}{\partial x_{1}}, \ldots, \frac{\partial V(x, t, i)}{\partial x_{n}}\right), \\
V_{x x}(x, t, i)=\left(\frac{\partial^{2} V(x, t, i)}{\partial x_{i} \partial x_{j}}\right)_{n \times n} .
\end{gathered}
$$

For the reader's convenience we cite the generalized Itô formula (see Skorohod 1989): if $V \in C^{2,1}\left(\mathbb{R}^{n} \times \mathbb{R}_{+} \times S ; \mathbb{R}_{+}\right)$, then for any stopping times $0 \leqslant \rho_{1}<\rho_{2}<\infty$,

$$
\mathrm{E} V\left(x\left(\rho_{2}\right), \rho_{2}, r\left(\rho_{2}\right)\right)=\mathrm{E} V\left(x\left(\rho_{1}\right), \rho_{1}, r\left(\rho_{1}\right)\right)+\mathrm{E} \int_{\rho_{1}}^{\rho_{2}} L V(x(s), x(s-\tau), s, r(s)) \mathrm{d} s
$$

as long as the expectations of the integrals exist. Let us point out that in the following whenever we apply this generalized formula the expectations of integrals involved do exist due to Theorem 2.1 and the conditions to be imposed.

\section{Moment exponential stability of linear delay equations}

From now on we shall discuss exponential stability. Let us start with the linear stochastic differential delay equation with Markovian switching of the form

$$
\mathrm{d} x(t)=[A(r(t)) x(t)+B(r(t)) x(t-\tau)] \mathrm{d} t+\sum_{k=1}^{m}\left[C_{k}(r(t)) x(t)+D_{k}(r(t)) x(t-\tau)\right] \mathrm{d} w_{k}(t)
$$

on $t \geqslant 0$, with initial data $x_{0}=\xi \in C_{\mathscr{F}_{0}}^{\mathrm{b}}\left([-\tau, 0] ; \mathbb{R}^{n}\right)$. We shall simply write

$$
A(i)=A_{i}, B(i)=B_{i}, C_{k}(i)=C_{k i}, D_{k}(i)=D_{k i},
$$

which are all $n \times n$ matrices. By Theorem 2.1, equation (3.1) has a unique global solution which is denoted by $x(t ; \xi)$ in this paper. Clearly, $(3.1)$ admits a trivial solution $x(t ; 0) \equiv 0$.

Theorem 3.1. Assume that there are symmetric positive definite matrices $Q_{i}, 1 \leqslant i \leqslant N$, such that all the $2 n \times 2 n$ matrices

$$
H_{i}=\left[\begin{array}{cc}
Q_{i} A_{i}+A_{i}^{\mathrm{T}} Q_{i}+I+\sum_{k=1}^{m} C_{k i}^{\mathrm{T}} Q_{i} C_{k i}+\sum_{j=1}^{N} \gamma_{i j} Q_{j}, & Q_{i} B_{i}+\sum_{k=1}^{m} C_{k i}^{\mathrm{T}} Q_{i} D_{k i} \\
B_{i}^{\mathrm{T}} Q_{i}+\sum_{k=1}^{m} D_{k i}^{\mathrm{T}} Q_{i} C_{k i}, & -I+\sum_{k=1}^{m} D_{k i}^{\mathrm{T}} Q_{i} D_{k i}
\end{array}\right]
$$

are negative definite, where $I$ is the $n \times n$ identity matrix. Then

$$
\limsup _{t \rightarrow \infty} \frac{1}{t} \log \left(\mathrm{E}|x(t ; \xi)|^{2}\right) \leqslant-\gamma
$$


for all $\xi \in C_{\mathscr{F}_{0}}^{\mathrm{b}}\left([-\tau, 0] ; \mathbb{R}^{n}\right)$, where $\gamma>0$ is the unique root of the equation

$$
\gamma\left(\lambda_{Q}+\tau \mathrm{e}^{\gamma \tau}\right)=\lambda_{H}+\left[1 \wedge\left(\lambda_{H} \mathrm{e}^{\gamma \tau}\right)\right]
$$

with

$$
\lambda_{Q}=\max _{1 \leqslant i \leqslant N} \lambda_{\max }\left(Q_{i}\right) \quad \text { and } \quad \lambda_{H}=\min _{1 \leqslant i \leqslant N}\left[-\lambda_{\max }\left(H_{i}\right)\right]
$$

In other words, the trivial solution of (3.1) is mean square exponentially stable and the second moment Lyapunov exponent should not be greater than $-\gamma$.

Proof. Note that $-1 \leqslant \lambda_{\max }\left(H_{i}\right)<0$ and $0<\lambda_{H} \leqslant 1$. It is then easy to see that (3.4) has a unique root $\gamma>0$. Fix any $\xi \in C_{\mathscr{T}_{0}}^{\mathrm{b}}\left([-\tau, 0] ; \mathbb{R}^{n}\right)$ and write $x(t ; \xi)=x(t)$. Define $V(x, t, i) \in C^{2,1}\left(\mathbb{R}^{n} \times \mathbb{R}_{+} \times S ; \mathbb{R}_{+}\right)$by

$$
V(x, t, i)=\mathrm{e}^{\gamma t}\left[x^{\mathrm{T}} Q_{i} x+\int_{t-\tau}^{t} \mathrm{E}|x(s)|^{2} \mathrm{~d} s\right] .
$$

By Itô's formula, we can derive that

$$
\begin{aligned}
\mathrm{E} V(x(t), t, r(t))= & \mathrm{E} V(x(0), 0, r(0))+\mathrm{E} \int_{0}^{t} L V(x(s), x(s-\tau), s, r(s)) \mathrm{d} s \\
= & \mathrm{E} V(x(0), 0, r(0))+\mathrm{E} \int_{0}^{t} \mathrm{e}^{\gamma s}\left[\gamma x^{\mathrm{T}}(s) Q_{i} x(s)+\gamma \int_{s-\tau}^{s}|x(\theta)|^{2} \mathrm{~d} \theta\right. \\
& \left.+\left(x^{\mathrm{T}}(s), x^{\mathrm{T}}(s-\tau)\right) H_{r(s)}\left(\begin{array}{c}
x(s) \\
x(s-\rho)
\end{array}\right)\right] \\
\leqslant & \left(\lambda_{Q}+\tau\right) \mathrm{E}\|\xi\|^{2}+\left(\gamma \lambda_{Q}-\lambda_{H}\right) \mathrm{E} \int_{0}^{t} \mathrm{e}^{\gamma s}|x(s)|^{2} \mathrm{~d} s \\
& +\gamma \mathrm{E} \int_{0}^{t} \mathrm{e}^{\gamma s} \int_{s-\tau}^{s}|x(\theta)|^{2} \mathrm{~d} \theta \mathrm{d} s-\lambda_{H} \mathrm{E} \int_{0}^{t} \mathrm{e}^{\gamma s}|x(s-\tau)|^{2} \mathrm{~d} s .
\end{aligned}
$$

Compute

$$
\begin{aligned}
\int_{0}^{t} \mathrm{e}^{\gamma s} \int_{s-\tau}^{s}|x(\theta)|^{2} \mathrm{~d} \theta \mathrm{d} s & =\int_{-\tau}^{t}|x(\theta)|^{2}\left(\int_{\theta \vee 0}^{(\theta+\tau) \wedge t} \mathrm{e}^{\gamma s} \mathrm{~d} s\right) \mathrm{d} \theta \\
& \leqslant \int_{-\tau}^{t}|x(\theta)|^{2} \tau \mathrm{e}^{\gamma(\theta+\tau)} \mathrm{d} \theta \leqslant \tau \mathrm{e}^{\gamma \tau}\left(\frac{\|\xi\|^{2}}{\gamma}+\int_{0}^{t} \mathrm{e}^{\gamma s}|x(s)|^{2} \mathrm{~d} s\right) .
\end{aligned}
$$

Also 


$$
\begin{aligned}
-\lambda_{H} \mathrm{E} \int_{0}^{t} \mathrm{e}^{\gamma s}|x(s-\tau)|^{2} \mathrm{~d} s & =-\lambda_{H} \mathrm{e}^{\gamma \tau} \mathrm{E} \int_{-\tau}^{t-\tau} \mathrm{e}^{\gamma s}|x(s)|^{2} \mathrm{~d} s \\
& \leqslant-\left[1 \wedge\left(\lambda_{H} \mathrm{e}^{\gamma \tau}\right)\right] \mathrm{E} \int_{0}^{t-\tau} \mathrm{e}^{\gamma s}|x(s)|^{2} \mathrm{~d} s \\
& \leqslant-\left[1 \wedge\left(\lambda_{H} \mathrm{e}^{\gamma \tau}\right)\right] \mathrm{E} \int_{0}^{t} \mathrm{e}^{\gamma s}|x(s)|^{2} \mathrm{~d} s+\mathrm{E} \int_{t-\tau}^{t} \mathrm{e}^{\gamma s}|x(s)|^{2} \mathrm{~d} s
\end{aligned}
$$

Substituting (3.9) and (3.10) into (3.8) and using (3.4), we obtain that

$$
\mathrm{E} V(x(t), t, r(t)) \leqslant\left[\lambda_{Q}+\tau\left(1+\mathrm{e}^{\gamma \tau}\right)\right] \mathrm{E}\|\xi\|^{2}+\mathrm{E} \int_{t-\tau}^{t} \mathrm{e}^{\gamma s}|x(s)|^{2} \mathrm{~d} s .
$$

On the other hand,

$$
\mathrm{E} V(x(t), t, r(t)) \geqslant \mathrm{e}^{\gamma t} \lambda_{q} \mathrm{E}|x(t)|^{2}+\mathrm{E} \int_{t-\tau}^{t} \mathrm{e}^{\gamma s}|x(s)|^{2} \mathrm{~d} s,
$$

where $\lambda_{q}=\min _{1 \leqslant i \leqslant N} \lambda_{\min }\left(Q_{i}\right)$. Combining (3.11) with (3.12) and using (3.6), we have

$$
\mathrm{e}^{\gamma t} \lambda_{q} \mathrm{E}|x(t)|^{2} \leqslant\left[\lambda_{Q}+\tau\left(1+\mathrm{e}^{\gamma \tau}\right)\right] \mathrm{E}\|\xi\|^{2}
$$

and the required assertion (3.3) follows. The proof is complete.

Corollary 3.2. Let $\lambda_{i}=\lambda_{\max }\left(A_{i}+A_{i}^{\mathrm{T}}\right)$ for $1 \leqslant i \leqslant N$. Assume that there exist $N$ positive constants $q_{i}$ such that

$$
1+q_{i}\left(\lambda_{i}+\left\|B_{i}\right\|+\sum_{k=1}^{m}\left[\left\|\mathrm{C}_{k i}^{\mathrm{T}} D_{k i}\right\|+\left\|C_{k i}\right\|^{2}\right]\right)+\sum_{j=1}^{N} \gamma_{i j} q_{j}<0
$$

and

$$
-1+q_{i}\left(\left\|B_{i}\right\|+\sum_{k=1}^{m}\left[\left\|C_{k i}^{\mathrm{T}} D_{k i}\right\|+\left\|D_{k i}\right\|^{2}\right]\right)<0
$$

for all $1 \leqslant i \leqslant N$. Then the trivial solution of equation (3.1) is mean square exponentially stable.

Proof. By choosing $Q_{i}=q_{i} I, H_{i}$ defined by (3.2) becomes

$$
H_{i}=\left[\begin{array}{cc}
q_{i}\left(A_{i}+A_{i}^{\mathrm{T}}\right)+I+q_{i} \sum_{k=1}^{m} C_{k i}^{\mathrm{T}} C_{k i}+\sum_{j=1}^{N} \gamma_{i j} q_{j} I, & q_{i} B_{i}+q_{i} \sum_{k=1}^{m} C_{k i}^{\mathrm{T}} D_{k i} \\
q_{i} B_{i}^{\mathrm{T}}+q_{i} \sum_{k=1}^{m} D_{k i}^{\mathrm{T}} C_{k i}, & -I+q_{i} \sum_{k=1}^{m} D_{k i}^{\mathrm{T}} D_{k i}
\end{array}\right] .
$$

For any $x, y \in \mathbb{R}^{n}$, compute 


$$
\begin{aligned}
\left(x^{\mathrm{T}}, y^{\mathrm{T}}\right) H_{i}\left(\begin{array}{c}
x \\
y
\end{array}\right) & x^{\mathrm{T}}\left(q_{i}\left(A_{i}+A_{i}^{\mathrm{T}}\right)+I+q_{i} \sum_{k=1}^{m} C_{k i}^{\mathrm{T}} C_{k i}+\sum_{j=1}^{N} \gamma_{i j} q_{j} I\right) x \\
& +2 q_{i} x^{\mathrm{T}}\left(B_{i}+\sum_{k=1}^{m} C_{k i}^{\mathrm{T}} D_{k i}\right) y+y^{\mathrm{T}}\left(-I+q_{i} \sum_{k=1}^{m} D_{k i}^{\mathrm{T}} D_{k i}\right) y \\
\leqslant & \left(q_{i} \lambda_{i}+1+q_{i} \sum_{k=1}^{m}\left\|C_{k i}\right\|^{2}+\sum_{j=1}^{N} \gamma_{i j} q_{j}\right)|x|^{2} \\
& +q_{i}\left(\left\|B_{i}\right\|+\sum_{k=1}^{m}\left\|C_{k i}^{\mathrm{T}} D_{k i}\right\|\right)\left(|x|^{2}+|y|^{2}\right)+\left(-1+q_{i} \sum_{k=1}^{m}\left\|D_{k i}\right\|^{2}\right)|y|^{2} \\
= & {\left[1+q_{i}\left(\lambda_{i}+\left\|B_{i}\right\|+\sum_{k=1}^{m}\left[\left\|C_{k i}^{\mathrm{T}} D_{k i}\right\|+\left\|C_{k i}\right\|^{2}\right]\right)+\sum_{j=1}^{N} \gamma_{i j} q_{j}\right]|x|^{2} } \\
& +\left[-1+q_{i}\left(\left\|B_{i}\right\|+\sum_{k=1}^{m}\left[\left\|C_{k i}^{\mathrm{T}} D_{k i}\right\|+\left\|D_{k i}\right\|^{2}\right]\right)\right]|y|^{2} .
\end{aligned}
$$

By conditions (3.13) and (3.14), $H_{i}$ is negative definite. The assertion of the corollary now follows from Theorem 3.1. The proof is complete.

It is easier to apply Corollary 3.2 than Theorem 3.1 since one needs only to find $N$ positive numbers $q_{i}$ instead of $N$ symmetric positive definite matrices. But it is still difficult to find such $q_{i}$ sometimes. We shall now establish a criterion, which is easy to verify, for the existence of such $q_{i}$ and hence for the mean square exponential stability.

Let us introduce some new notation. Let $B$ be a vector or matrix. By $B \geqslant 0$ we mean that each element of $B$ is non-negative. By $B>0$ we mean $B \geqslant 0$ and at least one element of $B$ is positive. By $B \gg 0$ we mean all elements of $B$ are positive. Let $B_{1}$ and $B_{2}$ be two vectors or matrices with same dimensions. We write $B_{1} \geqslant B_{2}, B_{1}>B_{2}$ and $B_{1} \gg B_{2}$ if and only if $B_{1}-B_{2} \geqslant 0, B_{1}-B_{2}>0$ and $B_{1}-B_{2} \gg 0$, respectively. Define

$$
\Theta=\left[\begin{array}{c}
\theta_{1}^{-1} \\
\theta_{2}^{-1} \\
\vdots \\
\theta_{N}^{-1}
\end{array}\right], \quad \vec{q}=\left[\begin{array}{c}
q_{1} \\
q_{2} \\
\vdots \\
q_{N}
\end{array}\right], \quad A=\operatorname{diag}\left(\rho_{1}, \rho_{2}, \ldots, \rho_{N}\right)-\Gamma,
$$

where

$$
\theta_{i}=\left\|B_{i}\right\|+\sum_{k=1}^{m}\left[\left\|C_{k i}^{\mathrm{T}} D_{k i}\right\|+\left\|D_{k i}\right\|^{2}\right]
$$




$$
\rho_{i}=-\lambda_{i}-\left\|B_{i}\right\|-\sum_{k=1}^{m}\left[\left\|\mathrm{C}_{k i}^{\mathrm{T}} D_{k i}\right\|+\left\|C_{k i}\right\|^{2}\right]
$$

and $\theta_{i}^{-1}=\infty$ if $\theta_{i}=0$. We can then write (3.13) and (3.14) as

$$
\Theta \gg \vec{q} \gg 0 \text { and } A \vec{q} \gg \overrightarrow{1}:=(1,1, \ldots, 1)^{\mathrm{T}} .
$$

The following corollary describes the sufficient conditions which guarantee the existence of the $q_{i} \mathrm{~s}$ and hence the mean square exponential stability of the trivial solution of (3.1).

Corollary 3.3. Assume that $A$ is inverse-positive, that is, $A^{-1}$ exists and $A^{-1}>0$. If

$$
\Theta \gg A^{-1} \overrightarrow{1}
$$

then the trivial solution of (3.1) is exponentially stable in mean square.

Proof. Note that $A^{-1} \overrightarrow{1} \gg 0$ since each row of $A^{-1}$ has all non-negative elements and has at least one positive element, that is, each row $>0$. By (3.17), we can choose a constant $\beta>1$ sufficiently close to 1 for

$$
\Theta \gg \beta A^{-1} \overrightarrow{1} \text {. }
$$

Set $\vec{q}=\beta A^{-1} \overrightarrow{1}$. Then $\Theta \gg \vec{q} \gg 0$. Moreover,

$$
A \vec{q}=\beta \overrightarrow{1} \gg \overrightarrow{1} .
$$

In other words, we can find $\vec{q}=\left(q_{1}, \ldots, q_{N}\right)^{\mathrm{T}}$ which satisfies (3.16), that is, (3.13) and (3.14). Hence, by Corollary 3.2, the trivial solution of (3.1) is exponentially stable in mean square. The proof is complete.

It is useful to point out that the matrix $A$ defined by (3.15) has non-positive off-diagonal entries. That is, if we adopt here the traditional notation by letting

$$
Z^{N \times N}=\left\{B=\left(b_{i j}\right) \in \mathbb{R}^{N \times N}: b_{i j} \leqslant 0, i \neq j\right\}
$$

then $A \in Z^{N \times N}$. It is known that $A \in Z^{N \times N}$ is inverse-positive if and only if $A$ is a nonsingular so-called M-matrix. Berman and Plemmons (1994) has an excellent discussion on M-matrices and lists many equivalent conditions. For example, $A$ is a non-singular M-matrix if and only if all the leading principal minors of $A$ are positive.

\section{Moment exponential stability of nonlinear delay equations}

Let us now return to the general delay equation (2.1), namely

$$
\mathrm{d} x(t)=f(x(t), x(t-\tau), t, r(t)) \mathrm{d} t+g(x(t), x(t-\tau), t, r(t)) \mathrm{d} w(t)
$$

on $t \geqslant 0$, with initial data $x_{0}=\xi \in C_{\mathscr{F}_{0}}^{\mathrm{b}}\left([-\tau, 0] ; \mathbb{R}^{n}\right)$. The solution of this equation is denoted by $x(t ; \xi)$ again. For the purpose of stability we may assume, without loss of 
generality, that $f(0,0, t, i) \equiv 0$ and $g(0,0, t, i) \equiv 0$. So (4.1) admits a trivial solution $x(t ; 0) \equiv 0$.

Theorem 4.1. Let (H1) hold. Let $p, c_{1}, c_{2}$ be positive numbers and $\lambda_{1}>\lambda_{2} \geqslant 0$. Assume that there exists a function $V(x, t, i) \in C^{2,1}\left(\mathbb{R}^{n} \times \mathbb{R}_{+} \times S ; \mathbb{R}_{+}\right)$such that

$$
c_{1}|x|^{p} \leqslant V(x, t, i) \leqslant c_{2}|x|^{p}
$$

for all $(x, t, i) \in \mathbb{R}^{n} \times \mathbb{R}_{+} \times S$, and

$$
L V(x, y, t, i) \leqslant-\lambda_{1}|x|^{p}+\lambda_{2}|y|^{p}
$$

for all $(x, y, t, i) \in \mathbb{R}^{n} \times \mathbb{R}^{n} \times \mathbb{R}_{+} \times S$. Then

$$
\limsup _{t \rightarrow \infty} \frac{1}{t} \log \left(\mathrm{E}|x(t ; \xi)|^{p}\right) \leqslant-\gamma
$$

for all $\xi \in C_{\mathscr{F}_{0}}^{\mathrm{b}}\left([-\tau, 0] ; \mathbb{R}^{n}\right)$, where $\gamma>0$ is the unique root to the equation

$$
\gamma\left(c_{2}+\tau \lambda_{2} \mathrm{e}^{\gamma \tau}\right)=\lambda_{1}-\lambda_{2}
$$

In other words, the trivial solution of (4.1) is pth moment exponentially stable and the pth moment Lyapunov exponent is not greater than $-\gamma$.

Proof. Fix any $\xi \in C_{\mathscr{F}_{0}}^{\mathrm{b}}\left([-\tau, 0] ; \mathbb{R}^{n}\right)$ and write $x(t ; \xi)=x(t)$. Define $U(x, t, i) \in$ $C^{2,1}\left(\mathbb{R}^{n} \times \mathbb{R}_{+} \times S ; \mathbb{R}_{+}\right)$by

$$
U(x, t, i)=\mathrm{e}^{\gamma t}\left[V(x, t, i)+\lambda_{2} \int_{t-\tau}^{t} \mathrm{E}|x(s)|^{p} \mathrm{~d} s\right] .
$$

By Itô's formula, we can derive that

$$
\begin{aligned}
\mathrm{E} U(x(t), t, r(t))= & \mathrm{E} U(x(0), 0, r(0))+\mathrm{E} \int_{0}^{t} L U(x(s), x(s-\tau), s, r(s)) \mathrm{d} s \\
= & \mathrm{E} U(x(0), 0, r(0))+\mathrm{E} \int_{0}^{t} \mathrm{e}^{\gamma s}\left[\gamma V(x(s), s, r(s))+\gamma \lambda_{2} \int_{s-\tau}^{s}|x(\theta)|^{p} \mathrm{~d} \theta\right. \\
& \left.+\lambda_{2}\left(|x(s)|^{p}-|x(s-\tau)|^{p}\right)+L V(x(s), x(s-\tau), s, r(s))\right] \mathrm{d} s \\
\leqslant & \left(c_{2}+\tau \lambda_{2}\right) \mathrm{E}\|\xi\|^{p}+\left(\gamma c_{2}+\lambda_{2}-\lambda_{1}\right) \mathrm{E} \int_{0}^{t} \mathrm{e}^{\gamma s}|x(s)|^{p} \mathrm{~d} s \\
& +\gamma \lambda_{2} \mathrm{E} \int_{0}^{t} \mathrm{e}^{\gamma s} \int_{s-\tau}^{s}|x(\theta)|^{p} \mathrm{~d} \theta \mathrm{d} s .
\end{aligned}
$$

Compute 


$$
\begin{aligned}
\mathrm{E} \int_{0}^{t} \mathrm{e}^{\gamma s} \int_{s-\tau}^{s}|x(\theta)|^{p} \mathrm{~d} \theta \mathrm{d} s & =\mathrm{E} \int_{-\tau}^{t}|x(\theta)|^{p}\left(\int_{\theta \vee 0}^{(\theta+\tau) \wedge t} \mathrm{e}^{\gamma s} \mathrm{~d} s\right) \mathrm{d} \theta \\
& \leqslant \mathrm{E} \int_{-\tau}^{t}|x(\theta)|^{p} \tau \mathrm{e}^{\gamma(\theta+\tau)} \mathrm{d} \theta \leqslant \tau \mathrm{e}^{\gamma \tau}\left(\frac{\mathrm{E}\|\xi\|^{p}}{\gamma}+\mathrm{E} \int_{0}^{t} \mathrm{e}^{\gamma s}|x(s)|^{p} \mathrm{~d} s\right)
\end{aligned}
$$

Substituting this into (4.7) and using (4.5), we obtain that

$$
\mathrm{E} U(x(t), t, r(t)) \leqslant\left[c_{2}+\tau \lambda_{2}\left(1+\mathrm{e}^{\gamma \tau}\right)\right] \mathrm{E}\|\xi\|^{p} .
$$

But

$$
\mathrm{E} U(x(t), t, r(t)) \geqslant c_{1} \mathrm{e}^{\gamma t} \mathrm{E}|x(t)|^{p} .
$$

Consequently

$$
\mathrm{E}|x(t)|^{p} \leqslant \frac{1}{c_{1}}\left[c_{2}+\tau \lambda_{2}\left(1+\mathrm{e}^{\gamma \tau}\right)\right] \mathrm{e}^{-\gamma t},
$$

and the required assertion (4.4) follows. The proof is complete.

\section{Almost sure exponential stability}

We now begin to discuss the almost sure exponential stability for the delay equation (4.1).

Theorem 5.1. Let (H1) hold. Assume that there is a constant $K>0$ such that for all $(x, y, t, i) \in \mathbb{R}^{n} \times \mathbb{R}^{n} \times \mathbb{R}_{+} \times S$,

$$
|f(x, y, t, i)| \vee|g(x, y, t, i)| \leqslant K(|x|+|y|) .
$$

Let $p>0$. Assume that the trivial solution of (4.1) is pth moment exponentially stable, that is, there is a positive constant $\gamma$ such that

$$
\limsup _{t \rightarrow \infty} \frac{1}{t} \log \left(\mathrm{E}|x(t ; \xi)|^{p}\right) \leqslant-\gamma
$$

for all $\xi \in C_{\mathscr{F}_{0}}^{\mathrm{b}}\left([-\tau, 0] ; \mathbb{R}^{n}\right)$. Then

$$
\limsup _{t \rightarrow \infty} \frac{1}{t} \log (|x(t ; \xi)|) \leqslant-\frac{\gamma}{p} \text { a.s. }
$$

In other words, pth moment exponential stability implies almost sure exponential stability.

Proof. The case when $p \geqslant 1$ without Markovian switching has been proved by Mao (1997); here we extend to the case when $p>0$ with Markovian switching. Fix the initial value $\xi$ arbitrarily and write $x(t ; \xi)=x(t)$. Let $\varepsilon \in(0, \gamma / 2)$ be arbitrary. By (5.2) and Theorem 2.1, 
there is a positive constant $\kappa$ such that

$$
\mathrm{E}|x(t)|^{p} \leqslant \kappa \mathrm{e}^{-(\gamma-\varepsilon) t} \quad \text { on } t \geqslant-\tau .
$$

Let $\bar{k}$ be an integer sufficiently large for

$$
(6 K)^{p}\left[(\tau / \bar{k})^{p}+C_{p}(\tau / \bar{k})^{p / 2}\right] \leqslant \frac{1}{2}\left(1 \wedge \mathrm{e}^{-\gamma \tau}\right),
$$

where $C_{p}$ is the constant given by the Burkholder-Davis-Gundy inequality (see Karatzas and Shreve 1991; or Mao 1997). Let $\sigma=\tau / \bar{k}$ and $k=\bar{k}+1, \bar{k}+2, \ldots$ Noting that for any $a, b, c \geqslant 0$,

$$
(a+b+c)^{p} \leqslant[3(a \vee b \vee c)]^{p}=3^{p}\left(a^{p} \vee b^{p} \vee c^{p}\right) \leqslant 3^{p}\left(a^{p}+b^{p}+c^{p}\right),
$$

we have that

$$
\begin{aligned}
\mathrm{E}\left[\sup _{(k-1) \sigma \leqslant t \leqslant k \sigma}|x(t)|^{p}\right] \leqslant & 3^{p} \mathrm{E}|x((k-1) \sigma)|^{p}+3^{p} \mathrm{E}\left(\int_{(k-1) \sigma}^{k \sigma}|f(x(s), x(s-\tau), s, r(s))| \mathrm{d} s\right)^{p} \\
& +3^{p} \mathrm{E}\left[\sup _{(k-1) \sigma \leqslant t \leqslant k \sigma}\left|\int_{(k-1) \sigma}^{t} g(x(s), x(s-\tau), s, r(s)) \mathrm{d} w(s)\right|^{p}\right]
\end{aligned}
$$

By (5.4),

$$
\mathrm{E}|x((k-1) \sigma)|^{p} \leqslant \kappa \mathrm{e}^{-(\gamma-\varepsilon)(k-1) \sigma} .
$$

Compute that

$$
\begin{aligned}
& \mathrm{E}\left(\int_{(k-1) \sigma}^{k \sigma}|f(x(s), x(s-\tau), s, r(s))| \mathrm{d} s\right)^{p} \\
& \quad \leqslant \mathrm{E}\left(\sigma \sup _{(k-1) \sigma \leqslant s \leqslant k \sigma}|f(x(s), x(s-\tau), s, r(s))|\right)^{p} \\
& \quad \leqslant(2 \sigma K)^{p} \mathrm{E}\left[\sup _{(k-1) \sigma \leqslant t \leqslant k \sigma}|x(t)|^{p}\right]+(2 \sigma K)^{p} \mathrm{E}\left[\sup _{(k-1) \sigma \leqslant t \leqslant k \sigma}|x(t-\tau)|^{p}\right] \\
& \quad \leqslant(2 \sigma K)^{p} \mathrm{E}\left[\sup _{(k-1) \sigma \leqslant t \leqslant k \sigma}|x(t)|^{p}\right]+(2 \sigma K)^{p} \mathrm{E}\left[\sup _{(k-1-\bar{k}) \sigma \leqslant t \leqslant(k-\bar{k}) \sigma}|x(t)|^{p}\right] .
\end{aligned}
$$

Compute also that 


$$
\begin{aligned}
& \mathrm{E}\left[\sup _{(k-1) \sigma \leqslant t \leqslant k \sigma}\left|\int_{(k-1) \sigma}^{t} g(x(s), x(s-\tau), s, r(s)) \mathrm{d} w(s)\right|^{p}\right] \\
& \quad \leqslant C_{p} \mathrm{E}\left(\int_{(k-1) \sigma}^{k \sigma}|g(x(s), x(s-\tau), s, r(s))|^{2} \mathrm{~d} s\right)^{p / 2} \\
& \quad \leqslant C_{p} \mathrm{E}\left(\sigma \sup _{(k-1) \sigma \leqslant s \leqslant k \sigma}|g(x(s), x(s-\tau), s, r(s))|^{2}\right)^{p / 2} \\
& \quad \leqslant C_{p}(2 K)^{p} \sigma^{p / 2} \mathrm{E}\left[\sup _{(k-1) \sigma \leqslant t \leqslant k \sigma}|x(t)|^{p}\right]+C_{p}(2 K)^{p} \sigma^{p / 2} \mathrm{E}\left[\sup _{(k-1-\bar{k}) \sigma \leqslant t \leqslant(k-\bar{k}) \sigma}|x(t)|^{p}\right] .
\end{aligned}
$$

Substituting (5.7)-(5.9) into (5.6) yields

$$
\begin{aligned}
\mathrm{E}\left[\sup _{(k-1) \sigma \leqslant t \leqslant k \sigma}|x(t)|^{p}\right] \leqslant & 3^{p} \kappa \mathrm{e}^{-(\gamma-\varepsilon)(k-1) \sigma}+(6 K)^{p}\left(\sigma^{p}+C_{p} \sigma^{p / 2}\right) \mathrm{E}\left[\sup _{(k-1) \sigma \leqslant t \leqslant k \sigma}|x(t)|^{p}\right] \\
& +(6 K)^{p}\left(\sigma^{p}+C_{p} \sigma^{p / 2}\right) \mathrm{E}\left[\sup _{(k-1-\bar{k}) \sigma \leqslant t \leqslant(k-\bar{k}) \sigma}|x(t)|^{p}\right] .
\end{aligned}
$$

Noting from (5.5) that $(6 K)^{p}\left(\sigma^{p}+C_{p} \sigma^{p / 2}\right) \leqslant 1 / 2$, we obtain from (5.10) that

$$
\begin{aligned}
& \mathrm{E}\left[\sup _{(k-1) \sigma \leqslant t \leqslant k \sigma}|x(t)|^{p}\right] \\
& \quad \leqslant 2 \kappa 3^{p} \mathrm{e}^{-(\gamma-\varepsilon)(k-1) \sigma}+2(6 K)^{p}\left(\sigma^{p}+C_{p} \sigma^{p / 2}\right) \mathrm{E}\left[\sup _{(k-1-\bar{k}) \sigma \leqslant t \leqslant(k-\bar{k}) \sigma}|x(t)|^{p}\right] \\
& \quad \leqslant 2 \kappa 3^{p} \mathrm{e}^{-(\gamma-\varepsilon)(k-1) \sigma}+\mathrm{e}^{-(\gamma-\varepsilon) \bar{k} \sigma} \mathrm{E}\left[\sup _{(k-1-\bar{k}) \sigma \leqslant t \leqslant(k-\bar{k}) \sigma}|x(t)|^{p}\right]
\end{aligned}
$$

where (5.5) has been used once again. Let $\rho=[k / \bar{k}$, the integer part of $k / \bar{k}$. By induction, we can show from $(5.11)$ that

$$
\mathrm{E}\left[\sup _{(k-1) \sigma \leqslant t \leqslant k \sigma}|x(t)|^{p}\right] \leqslant 2 \rho \kappa 3^{p} \mathrm{e}^{-(\gamma-\varepsilon)(k-1) \sigma}+\mathrm{e}^{-(\gamma-\varepsilon) \rho \bar{k} \sigma} \mathrm{E}\left[\sup _{(k-1-\rho \bar{k}) \sigma \leqslant t \leqslant(k-\rho \bar{k}) \sigma}|x(t)|^{p}\right] .
$$

Since $k / \bar{k}-1 \leqslant \rho \leqslant k / \bar{k}$, we have $k-\bar{k} \leqslant \rho \bar{k} \leqslant k$ and hence

$$
-\tau \leqslant-\sigma \leqslant(k-1-\rho \bar{k}) \sigma \leqslant(k-\rho \bar{k}) \sigma \leqslant \bar{k} \sigma=\tau .
$$

We therefore see from (5.12) that 


$$
\begin{aligned}
\mathrm{E}\left[\sup _{(k-1) \sigma \leqslant t \leqslant k \sigma}|x(t)|^{p}\right] & \leqslant \frac{2 k \kappa}{\bar{k}} 3^{p} \mathrm{e}^{-(\gamma-\varepsilon)(k-1) \sigma}+\mathrm{e}^{-(\gamma-\varepsilon)(k-\bar{k}) \sigma} \mathrm{E}\left[\sup _{-\tau \leqslant t \leqslant \tau}|x(t)|^{p}\right] \\
& \leqslant C(k+1) \mathrm{e}^{-(\gamma-\varepsilon) k \sigma},
\end{aligned}
$$

where

$$
C=\frac{2 \kappa}{\bar{k}} 3^{p} \mathrm{e}^{\gamma \sigma}+\mathrm{e}^{\gamma \bar{k} \sigma} \mathrm{E}\left[\sup _{-\tau \leqslant t \leqslant \tau}|x(t)|^{p}\right]
$$

and, by Theorem 2.1, $C<\infty$. Hence

$$
P\left\{\omega: \sup _{(k-1) \sigma \leqslant t \leqslant k \sigma}|x(t)|>\mathrm{e}^{-(\gamma-2 \varepsilon) k \sigma / p}\right\} \leqslant C(k+1) \mathrm{e}^{-\varepsilon k \sigma} .
$$

In view of the well-known Borel-Cantelli lemma, we see that for almost all $\omega \in \Omega$,

$$
\sup _{(k-1) \sigma \leqslant t \leqslant k \sigma}|x(t)| \leqslant \mathrm{e}^{-(\gamma-2 \varepsilon) k \sigma / p}
$$

holds for all but finitely many $k$. Hence there exists a $k_{0}(\omega)$, for all $\omega \in \Omega$ excluding a $P$-null set, for which (5.14) holds whenever $k \geqslant k_{0}$. Consequently, for almost all $\omega \in \Omega$,

$$
\frac{1}{t} \log (|x(t)|) \leqslant-\frac{(\gamma-2 \varepsilon) k \sigma}{p t} \leqslant-\frac{\gamma-2 \varepsilon}{p}
$$

if $(k-1) \sigma \leqslant t \leqslant k \sigma$. Therefore

$$
\limsup _{t \rightarrow \infty} \frac{1}{t} \log (|x(t)|) \leqslant-\frac{\gamma-2 \varepsilon}{p} \text { a.s. }
$$

and the required (5.3) follows by letting $\varepsilon \rightarrow 0$. The proof is complete.

\section{Examples}

In this Section we shall discuss three examples to illustrate our theory. In the following examples we shall omit mentioning the initial data.

Example 6.1. Consider a one-dimensional linear stochastic differential delay equation with Markovian switching of the form

$$
\mathrm{d} x(t)=\alpha(r(t)) x(t) \mathrm{d} t+\sigma(r(t)) x(t-\tau) \mathrm{d} w(t)
$$

on $t \geqslant 0$. Here the Markov chain $r(t)$ is the same as before, but $w(t)$ is a scalar Brownian motion independent of $r(t), \alpha(i)$ and $\sigma(i)$ are all constants and we shall write $\alpha(i)=\alpha_{i}$ and $\sigma(i)=\sigma_{i}$. To apply Corollary 3.3, we note that $\Theta$ and $A$ defined by (3.15) become 


$$
\Theta=\left[\begin{array}{c}
\sigma_{1}^{-2} \\
\sigma_{2}^{-2} \\
\vdots \\
\sigma_{N}^{-2}
\end{array}\right] \text { and } A=\operatorname{diag}\left(-2 \alpha_{1},-2 \alpha_{2}, \ldots,-2 \alpha_{N}\right)-\Gamma
$$

where $\sigma_{i}^{-2}=\infty$ if $\sigma_{i}=0$. By Corollary 3.3, if $A^{-1}>0$ and

$$
\Theta \gg A^{-1} \overrightarrow{1}
$$

then the trivial solution of (6.1) is exponentially stable in mean square. Moreover, by Theorem 5.1, the trivial solution is also almost surely exponentially stable. It is interesting to note that (6.2) holds if all $\sigma_{i}=0$. We therefore observe that if $A^{-1}>0$, then the trivial solution of the delay equation

$$
\frac{\mathrm{d} x(t)}{\mathrm{d} t}=\alpha(r(t)) x(t)
$$

is exponentially stable in mean square. Moreover, equation (6.1) can be regarded as the stochastically perturbed system of (6.3), while condition (6.2) means that if the intensity $\sigma_{i}$ of the stochastic perturbation is sufficiently small then the perturbed system (6.1) will remain stable.

Example 6.2. Let $w(t)$ be a scalar Brownian motion. Let $r(t)$ be a right-continuous Markov chain taking values in $S=\{1,2,3\}$ with generator

$$
\Gamma=\left[\begin{array}{ccc}
-2 & 1 & 1 \\
3 & -4 & 1 \\
1 & 1 & -2
\end{array}\right]
$$

Assume that $w(t)$ and $r(t)$ are independent. Consider a three-dimensional linear stochastic differential delay equation with Markovian switching of the form

$$
\mathrm{d} x(t)=A(r(t)) x(t) \mathrm{d} t+D(r(t)) x(t-\tau) \mathrm{d} w(t)
$$

on $t \geqslant 0$, where

$$
\begin{gathered}
A(1)=A_{1}=\left[\begin{array}{ccc}
-2 & 1 & -2 \\
2 & -2 & 1 \\
1 & -2 & -3
\end{array}\right], \quad A(2)=A_{2}=\left[\begin{array}{ccc}
0.5 & 1 & 0.5 \\
-0.8 & 0.5 & 1 \\
-0.7 & -0.9 & 0.2
\end{array}\right], \\
A(3)=A_{3}=\left[\begin{array}{ccc}
-0.5 & -0.9 & -1 \\
1 & -0.6 & -0.7 \\
0.8 & 1 & -1
\end{array}\right],
\end{gathered}
$$

and $D(i)=D_{i}$ are all $3 \times 3$ constant matrices. To apply Corollary 3.3, we compute $\lambda_{i}=$ $\lambda_{\text {max }}\left(A_{i}+A_{i}^{\mathrm{T}}\right)$ :

$$
\lambda_{1}=-2.4385, \quad \lambda_{2}=1.20718, \quad \lambda_{3}=-0.95067 .
$$


The vector $\Theta$ and matrix $A$ defined by (3.15) become

$$
\Theta=\left(\left\|D_{1}\right\|^{-2}, \ldots,\left\|D_{N}\right\|^{2}\right)^{\mathrm{T}}
$$

and

$$
A=\operatorname{diag}(2.4385,-1.20718,0.95067)-\Gamma=\left[\begin{array}{ccc}
4.4385 & -1 & -1 \\
-3 & 2.79282 & -1 \\
-1 & -1 & 2.95067
\end{array}\right] .
$$

Compute

$$
A^{-1}=\left[\begin{array}{lll}
0.439017 & 0.239537 & 0.229966 \\
0.597346 & 0.733437 & 0.451010 \\
0.351230 & 0.329746 & 0.569693
\end{array}\right]
$$

and

$$
A^{-1}(1,1,1)^{\mathrm{T}}=(0.90852,1.78179,1.25067)^{\mathrm{T}} .
$$

Therefore, by Corollary 3.3, if $\Theta \gg A^{-1}(1,1,1)^{\mathrm{T}}$, that is,

$$
\left\|D_{1}\right\|^{2}<1.10069, \quad\left\|D_{2}\right\|^{2}<0.56123, \quad\left\|D_{3}\right\|^{2}<0.79957,
$$

then the trivial solution of equation (6.4) is exponentially stable in mean square. Moreover, by Theorem 5.1, it is also almost surely exponentially stable. As explained in Section 1, equation (6.4) can be regarded as the result of the following three stochastic differential delay equations

$$
\begin{aligned}
& \mathrm{d} x(t)=A_{1} x(t) \mathrm{d} t+D_{1} x(t-\tau) \mathrm{d} w(t), \\
& \mathrm{d} x(t)=A_{2} x(t) \mathrm{d} t+D_{2} x(t-\tau) \mathrm{d} w(t), \\
& \mathrm{d} x(t)=A_{3} x(t) \mathrm{d} t+D_{3} x(t-\tau) \mathrm{d} w(t)
\end{aligned}
$$

switching among each other according to the movement of the Markov chain. Note that (6.6b) is not exponentially stable in mean square (since $A_{2}+A_{2}^{\mathrm{T}}$ is positive definite) but (6.6a) is, while (6.6c) may be. However, as the result of Markovian switching, the overall behaviour, expressed by (6.4), is exponentially stable.

Example 6.3. Let $w(t)$ be a scalar Brownian motion. Let $r(t)$ be a right-continuous Markov chain taking values in $S=\{1,2\}$ with generator

$$
\Gamma=\left(\gamma_{i j}\right)_{2 \times 2}=\left[\begin{array}{cc}
-1 & 1 \\
1 & -1
\end{array}\right] .
$$

Assume that $w(t)$ and $r(t)$ are independent. Consider a one-dimensional stochastic differential delay equation with Markovian switching of the form

$$
\mathrm{d} x(t)=f(x(t), t, r(t)) \mathrm{d} t+g(x(t-\tau), t, r(t)) \mathrm{d} w(t)
$$


on $t \geqslant 0$, where $f, g: \mathbb{R} \times \mathbb{R}_{+} \times S \rightarrow \mathbb{R}$. Assume that

$$
x f(x, t, 1) \leqslant \frac{x^{2}}{4}, \quad x f(x, t, 2) \leqslant-3 x^{2}
$$

for all $(x, t) \in \mathbb{R} \times \mathbb{R}_{+}$, while

$$
|g(y, t, 1)| \leqslant \frac{|y|}{8}, \quad|g(y, t, 2)| \leqslant \frac{|y|}{4}
$$

for all $(y, t) \in \mathbb{R} \times \mathbb{R}_{+}$. To examine the third moment exponential stability, we construct a function $V: \mathbb{R} \times \mathbb{R}_{+} \times S \rightarrow \mathbb{R}_{+}$by

$$
V(x, t, i)=\beta_{i}|x|^{3},
$$

with $\beta_{2}=1$ and $\beta_{1}=\beta>0$ a constant to be determined. It is easy to show that the operator $L V$ from $\mathbb{R} \times \mathbb{R} \times \mathbb{R}_{+} \times S$ to $\mathbb{R}$ has the form

$$
L V(x, y, t, i)=3 \beta_{i}|x| x f(x, t, i)+3 \beta_{i}|x \| g(y, t, i)|^{2}+\left(\gamma_{i 1} \beta+\gamma_{i 2}\right)|x|^{3} .
$$

By conditions (6.8) and (6.9), we then have

$$
L V(x, y, t, i) \leqslant \begin{cases}-[0.25 \beta-1]|x|^{3}+\frac{3 \beta}{64}|x \| y|^{2} & \text { if } i=1, \\ -(10-\beta)|x|^{3}+\frac{3}{16}|x \| y|^{2} & \text { if } i=2 .\end{cases}
$$

Noting that

$$
|x||y|^{2} \leqslant \frac{1}{3}|x|^{3}+\frac{2}{3}|y|^{3}
$$

we obtain that

$$
L V(x, y, t, 1) \leqslant-(0.234375 \beta-1)|x|^{3}+0.03125 \beta|y|^{3}
$$

and

$$
L V(x, y, t, 2) \leqslant-(9.9375-\beta)|x|^{3}+0.125|y|^{3} .
$$

Choosing $\beta$ as the solution to

$$
0.234375 \beta-1=9.9375-\beta,
$$

that is, $\beta=8.8608$, we then have

$$
L V(x, y, t, i) \leqslant \begin{cases}-1.0767|x|^{3}+0.2769|y|^{3} & \text { if } i=1, \\ -1.0767|x|^{3}+0.125|y|^{3} & \text { if } i=2 .\end{cases}
$$

So

$$
L V(x, y, t, i) \leqslant-1.0767|x|^{3}+0.2769|y|^{3} .
$$

By Theorem 4.1, we conclude that the trivial solution of (6.7) is third moment exponentially stable and, moreover, the third moment Lyapunov exponent is not greater than the unique root $\gamma>0$ of the equation 


$$
\gamma\left(8.8608+0.2769 \tau \mathrm{e}^{\gamma \tau}\right)=0.7998 .
$$

For instance, the unique root of this equation is $\gamma=0.09$ if $\tau=0.1$.

\section{Acknowledgements}

The authors would like to thank the referees for their helpful suggestions and detailed remarks. The authors would also like to thank the Royal Society, the Biotechnology and Biological Sciences Research Council and the Engineering and Physical Sciences Research Council for their financial support.

\section{References}

Arnold, L. (1972) Stochastic Differential Equations: Theory and Applications. New York: Wiley.

Basak, G.K., Bisi, A. and Ghosh, M.K. (1996) Stability of a random diffusion with linear drift. $J$. Math. Anal. Appl., 202, 604-622.

Berman, A. and Plemmons, R.J. (1994) Nonnegative Matrices in the Mathematical Sciences. Philadelphia: Society of Industrial and Applied Mathematics.

Ghosh, M.K., Arapostathis, A. and Marcus, S.I. (1993) Optimal control of switching diffusions with application to flexible manufacturing systems. SIAM J. Control Optim., 31, 1183-1204.

Has'minskii, R.Z. (1981) Stochastic Stability of Differential Equations. Moscow: Sijthoff and Noordhoff.

Karatzas, I. and Shreve, S.E. (1991) Brownian Motion and Stochastic Calculus, 2nd edition. Berlin: Springer-Verlag.

Kolmanovskii, V.B. and Myshkis, A. (1992) Applied Theory of Functional Differential Equations. Boston: Kluwer Academic Publishers.

Kolmanovskii, V.B. and Nosov, V.R. (1986) Stability of Functional Differential Equations. London: Academic Press.

Ladde, G.S. and Lakshmikantham, V. (1980) Random Differential Inequalities. New York: Academic Press.

Mao, X. (1991) Stability of Stochastic Differential Equations with Respect to Semimartingales. Harlow: Longman Scientific and Technical.

Mao, X. (1994) Exponential Stability of Stochastic Differential Equations. New York: Marcel Dekker.

Mao, X. (1997) Stochastic Differential Equations and Applications. Chichester: Ellis Horwood.

Mohammed, S.-E.A. (1986) Stochastic Functional Differential Equations. Harlow: Longman Scientific and Technical.

Skorohod, A.V. (1989) Asymptotic Methods in the Theory of Stochastic Differential Equations. Providence, RI: American Mathematical Society.

Received December 1997 and revised July 1998 